\title{
Design and implementation of monitoring and warning (IOT) system for electricity poles
}

\author{
Jumana Amer AL-Hammoudi*, Basil H. Jasim
}

Electrical Engineering Department, University of Basrah, Basrah, Iraq

\author{
Correspondence \\ * Jumana Amer AL-Hammoudi \\ Electrical Engineering Department, \\ University of Basrah, Basrah, Iraq \\ Email: jumana.fnmr@yahoo.com
}

\begin{abstract}
There are many serious accidents on human life caused by electric current columns, and it is possible for the Internet of Things to find solutions to prevent the risks that occur, as in many fields such as medicine, agriculture, industry and others. In this paper, we will show monitoring and tracking of the current that passes through the electrical poles and the possible leakage, in addition to monitoring the temperature and humidity in the area, and knowing the condition of the light in the column according to morning and evening, this proposed system that will perform a general purpose added to the region. using Open source NODE MCU board, GPS positioning, current sensor, temperature and humidity sensor that provide desired data via open source platforms that we have chosen to be ThingSpeak that easily to handle.
\end{abstract}

KEYWORDS: IOT, Node MCU board, ACS712sensor, ThingSpeak, DHT11, GPS, LDR.

\section{INTRODUCTION}

A long time ago, the Internet of things (or the Internet of everything) appeared in the military fields, but with the development of technology and the increase in the use of the Internet and wireless technologies and the widespread use of mobile phones. The existence IOT became necessary to provide human services with high technology and quality. Cloud computing and IPV6 foster an integrated development between the Internet and IOT, as the Internet of Things acts as a huge network of objects that connect with each other and with the individual across the Internet by making each device associated with a single definition capable of collecting and processing information in real time individually. The entry of IPV6 with IOT has made it possible to mix an unknown number of things on the Internet [1][2]. In 1999 IOT was launched by Kevin Ashton, who dreams of a system in which anything physical can be connected to the Internet via sensors everywhere. In 2013, the Global Standards Initiative (GSI) determined that the Internet of Things was the infrastructure for the information society [3].

The Internet of Things has become a supporter of many areas in the process of connecting devices with sensors surrounding the Internet and facilitating the access to information, exchange and processing of required information anywhere in the world and at any time through ready-made software platforms from anywhere in the world via the Internet. The Internet of Things has made life and business faster and easier than it has been, as well as flexibility in dealing with devices and making people more free in their lives.

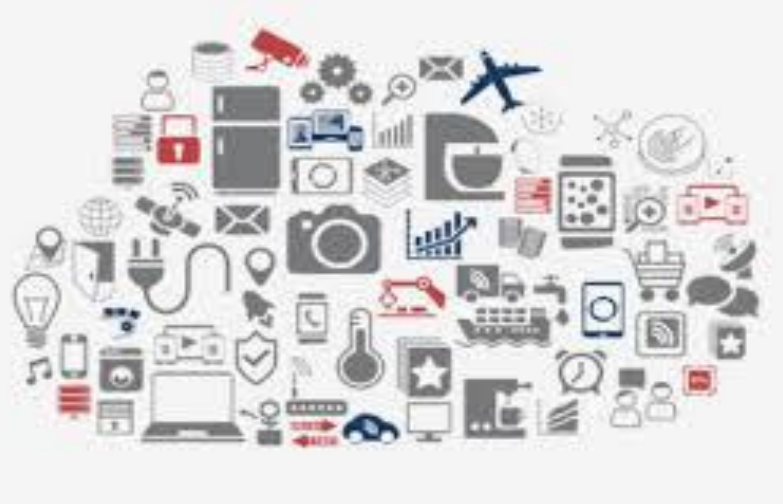

Fig. 1: Internet of thing (iot)

The rest of this paper is organized as follows. Section II introduces IOT applications, Section III presents the proposed system for monitoring. and warning IoT system for the electrical poles, while Section IV presents the hardware components used in system, Section V displays the software part and results, and finally, Section VI presents the concludes of the paper. 


\section{IOT APPLICATIONS}

The applications in the Internet of things appeared clearly over its ability to communicate with humans and devices, as we will present some of them:

In the literature [4] the researcher describes home appliances more intelligent with(IoT) through sensors and microcontrollers that collect and continuously transmit data via the Internet via phones / personal computers and process them remotely without the need to stick to the place and restrict human freedoms.

In the literature [5], the entry of the Internet has revealed things in the medical field when discussing cases of heart patients, as it monitors and follows patients through sensors and sends their data to the Internet to follow up on the matter by the specialist doctor and also gives treatment via the Internet, these techniques avoided many risks for the elderly and cases Fast, which can lead to the loss of the patient and thus be safer in his health.

The author in [6] describes the possibility of monitoring water quality and turbidity and controlling this in real time through sensors that send their data through the microcontrollers to the official in handling the matter through the Internet. What led to the Internet of things to have a role in finding a valid and self-sustaining environment High quality water and serve humans and living organisms in drinking water.

In this [7] research, the monitoring system used to distribute and transfer electrical energy is mentioned, at the present time, interest in introducing smart systems and devices to achieve comfortable purposes for life has become. The electric power sector has created what is known as a smart grid that works to offset the demand for electricity supplies and benefit from electrical resources. Effectively. The network monitors the distribution and transmission of energy and establishes several smart communications in the system to enhance energy services to make them more responsive, powerful and communicative.

Many developing countries, such as Indonesia, are working with a combination of wireless transmission with the ZigBee and GPRS to meet the basic requirements in terms of realtime communication and long distances for a monitoring system through the Internet of things that are displayed on the computer and even the mobile phone, and the development is still ongoing to design the power distribution system devices more effectively.

On this page [ 8] he talked about using the library system that enables it to continuously update data and information for employees and students without the need for the human factor. It was designed with GMS technology with a built-in server to send SMS to the user what he needs and requests. For the customer. He also made it possible to store previous notes and notifications that were sent.

In this proposed work, we will explain the process of monitoring and tracking electrical and leakage cases using the ACS712 sensor, as well as changes in the area in terms of humidity and heat using the DHT11 sensor and tracking the light used in the column next to the light sensor, using the NODE MCU controller board, in addition to GPS, and send all data to ThingSpeak online platform.

\section{PROPOSED SYSTEM}

In this paper, we proposed monitoring the current passing through the electrical poles and discovering the current leakage and tracking that in platform ThingSpeak site, so that get a warning from electrical leakage. the ACs712 sensor, that be sensing the current passed in poles and transmit data in real time to ThingSpeak. this propose will lead to find a solutions speed up to prevent danger from controller room. In addition to the presence of the DHT11 temperature and humidity sensor, which shows the temperature and humidity of the area, as we know its influence on the columns and wires, as the humidity increases, the probability of leakage will increase in the electrical column and that the high temperature affecting the state of the wires and their expansion, which will lead to merging and leakage cases. It will send the DHT11 sensor information to the platform.

With the addition of a light sensor that monitors the work of light at day and night during the day, as the data sent to the site indicates the state of light at the column, it becomes clear to us the safety of the light attached to the column. In this work, we have linked the GPS locator, which helps to facilitate the process of determining the leakage current, thus reducing much time and speeding up the processing task.

Where depended in this paper to find the problem only, using one of the microcontrollers that are characterized by their small size and low cost is NodeMCU and we will explain in the following paragraphs, where it is installed within the Arduino IDE environment working on it in the same well-known Arduino programming language. The following Fig. (2) shows the hardware connection proposed system.

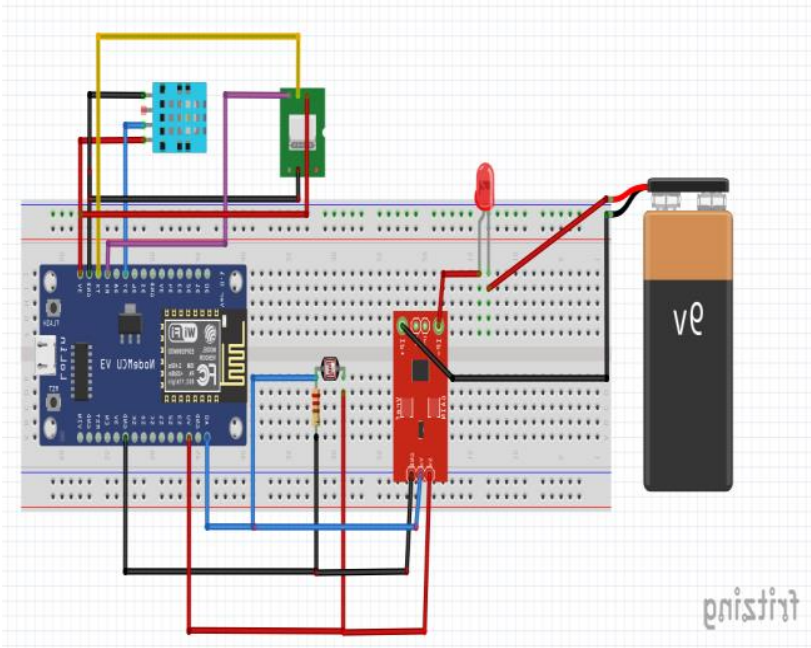

Fig. 2: Hardware connection system 
We notice in Fig. (2) that the NODE MCU board microcontroller will be the main board at work, and is considered to be the gateway between the sensors and the cloud computing that stores information sent via the Internet from the sensors. Fig. (3) show block diagram system

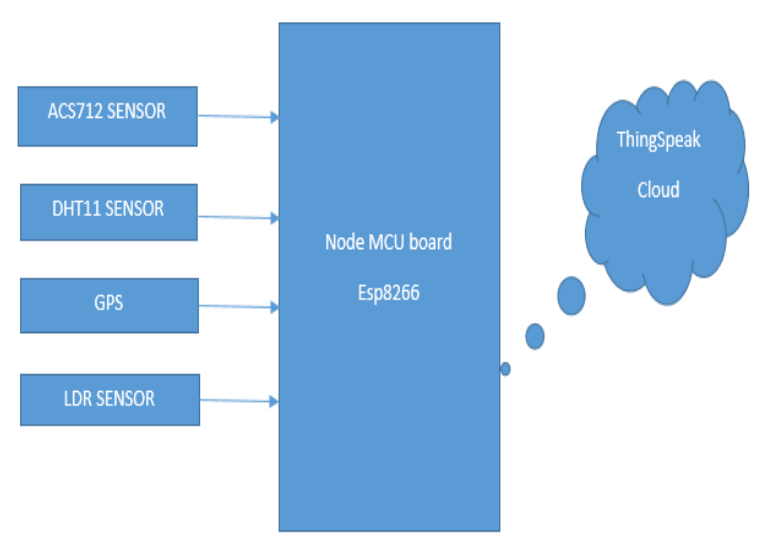

Fig. 3: block diagram for the system

\section{COMPONENTS REQUIRED}

\section{A. ACS712 Current Sensor}

The ACS712 provides economical and precise solutions for $\mathrm{AC}$ or DC current sensing in industrial, commercial, and communications systems. The device package allows for easy implementation by the customer. Typical applications include motor control, load detection and management, switched-mode power supplies, and overcurrent fault protection The ACS712 Current Sensors offered on the internet are designed to be easily used with micro controllers like the Arduino and other that. These sensors are based on the Allegro ACS712ELC chip. These current sensors are offered with full scale values of 5A, 20A and 30A [9].

the Fig. (4) below present the circuit connected with current sensor in the two poles and the other side connected with microcontroller NodeMCU have three pin, pin Vcc connect with PIN power, GND PIN, and PIN analog signal.

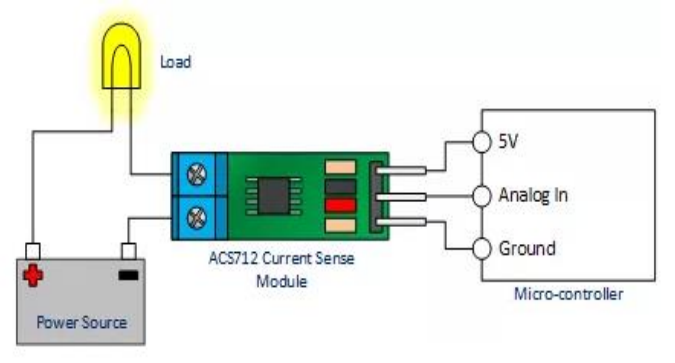

Fig. 4: circuit diagram current sensor

\section{B. GPS}

The NEO-6 module collection is a family of stand-alone GPS receivers providing excessive-overall performance u-blox 6 positioning engine. These bendy and fee-effective receivers offer a couple of connectivity alternatives in a miniature 16 x $12.2 \times 2.4 \mathrm{~mm}$ bundle. The compact architecture, energy and reminiscence alternatives make the NEO- 6 modules best for battery operated cell devices with limited price and area constraints. Innovative layout and era suppresses jamming sources and mitigates multipath consequences which provide NEO-6 GPS receivers first rate navigation overall performance even within the most difficult environments [10].

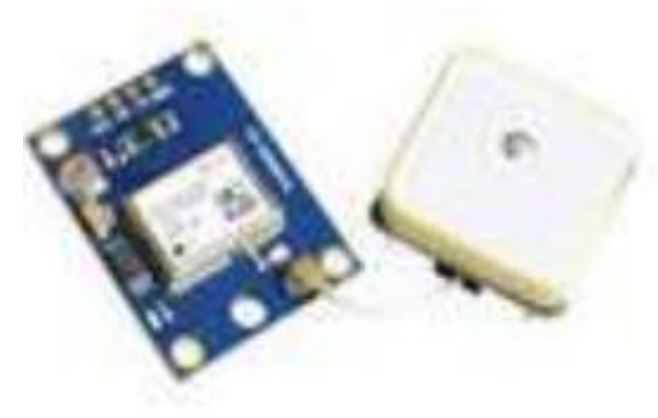

\section{LDR Sensor}

It is a component made up of highly resistant semiconductor materials or called photo conductors or photo cells only or optical cells. These resistors are

distinguished by their dependence on the light intensity they are exposed to, as the resistance decreases with increasing light intensity. This resistance entered in many areas because of its low cost and ease of use as it was in automatic street lights systems and in smart home systems and other light sensing circuits [11] [12]. In the proposed system for this page, as resistance sensitive to the dark and when it is darkness will be Turn on the lights and when there is light it will work opposite that.

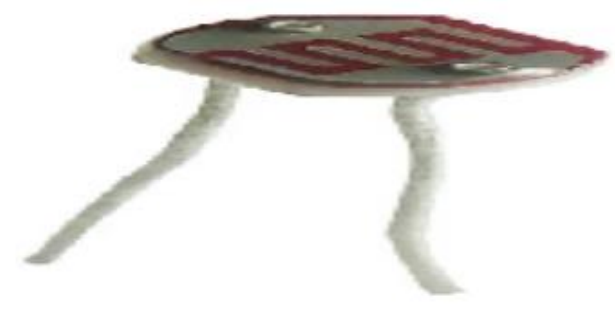

\section{Temperature and Humidity Sensor DHT11:}

It is a low-cost, easy-to-use digital sensor, it utilizes a capacitive humidity sensor and a thermistor to gauge the surrounding air, what is wrong with this sensor is its ability 
to give new information when every two seconds pass when using our library.it works on 3-5V power supply, Humidity ranges between $20-80 \%$ and heat ranges from $0-50{ }^{\circ} \mathrm{C}$ [13] [14].

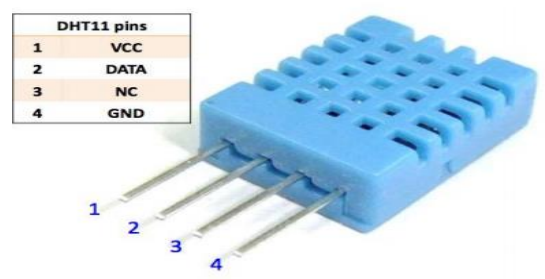

\section{E. NODE MCU Board}

NodeMCU board is an open source and can operate with in IDE Arduino. It consists of firmware which runs on ESP8266. It is programmable, low cost, and smart Wi-Fi enabled. NodeMCU has ESP-12 based serial Wi-

Fi integrated on-board to provide GPIO, PWM, ADC, I2C and built-in USB-TTL serial as shown in Fig. below. NodeMCU has powerful processing and storage capabilities that, allows the sensors to be integrated with it.it is Operating voltages (3.3-3.6V), Uses IPV4, HTTP, FTP, UDP \& TCP network protocols, operating frequency range $(2.4-2.6 \mathrm{GHz})$, Configured in both Android and iOS devices [15].

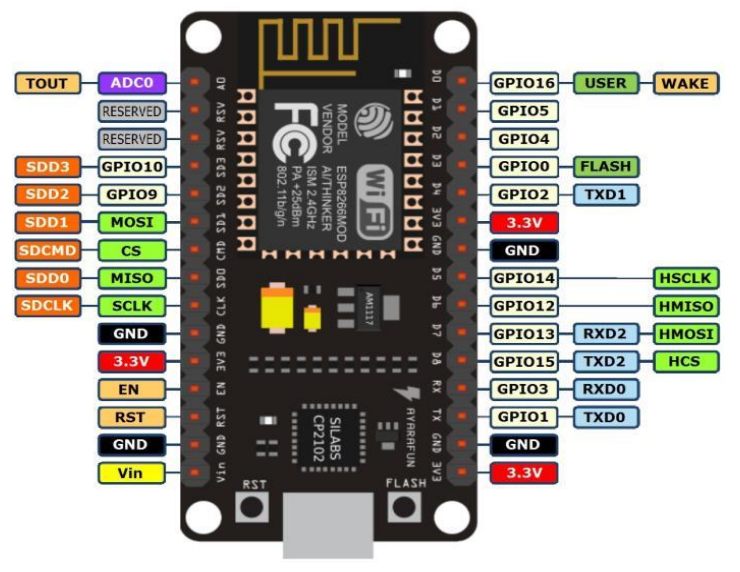

\section{V.SOFTWARE WORK AND RESULT}

\section{A. Thing Speak Platform}

ThingSpeak is an open source platform that appeared in 2010. It is used in many internet of things applications. It stores the data collected in real time from the sensors and stored inside the channels. It includes 4 channels and each channel allows to store up to 8 data fields, using up to 255 characters each. There are also 4 fields dedicated to the data on the site, and it consists of: description, length, length, height. Also, each channel can be public for all users and display its information without requiring a key, but when you make it private, the channel will give you a writing key and read key to make it private, it features a very easy visualization of the data collected from the sensors using simple and easy graphics.

ThingSpeak has the ability to integrate with small controls like Arduino, Raspberry Pi and others. ThingSpeak source includes HTTP request processing, data storage (alphanumeric), digital data processing, site tracking, and status updates [16] [17].

\section{B. Getting Start}

At the beginning of the worked, we will define the microcontroller that we are dealing with it, as our worked will be linked to the Internet. We choose a microcontroller with Wi-Fi inside it to make work easier, it's Nodemcu. Initially, we will define NodeMCU within the Arduino environment to allow us to work within the environment and download the code on it, and the steps to defined it inside the Arduino environment itself with any other microcontroller. Now in IDE environment file > Preferences> Additional URL Fields for board Manager: (https://arduino.esp8266.com/stable/package_esp8266com_ index.json).Then Tools> board > board manager, search about esp8266 and install it. After finishing, we find in the boards all esp8266 boards - includes it NodeMCU and its various versions. 


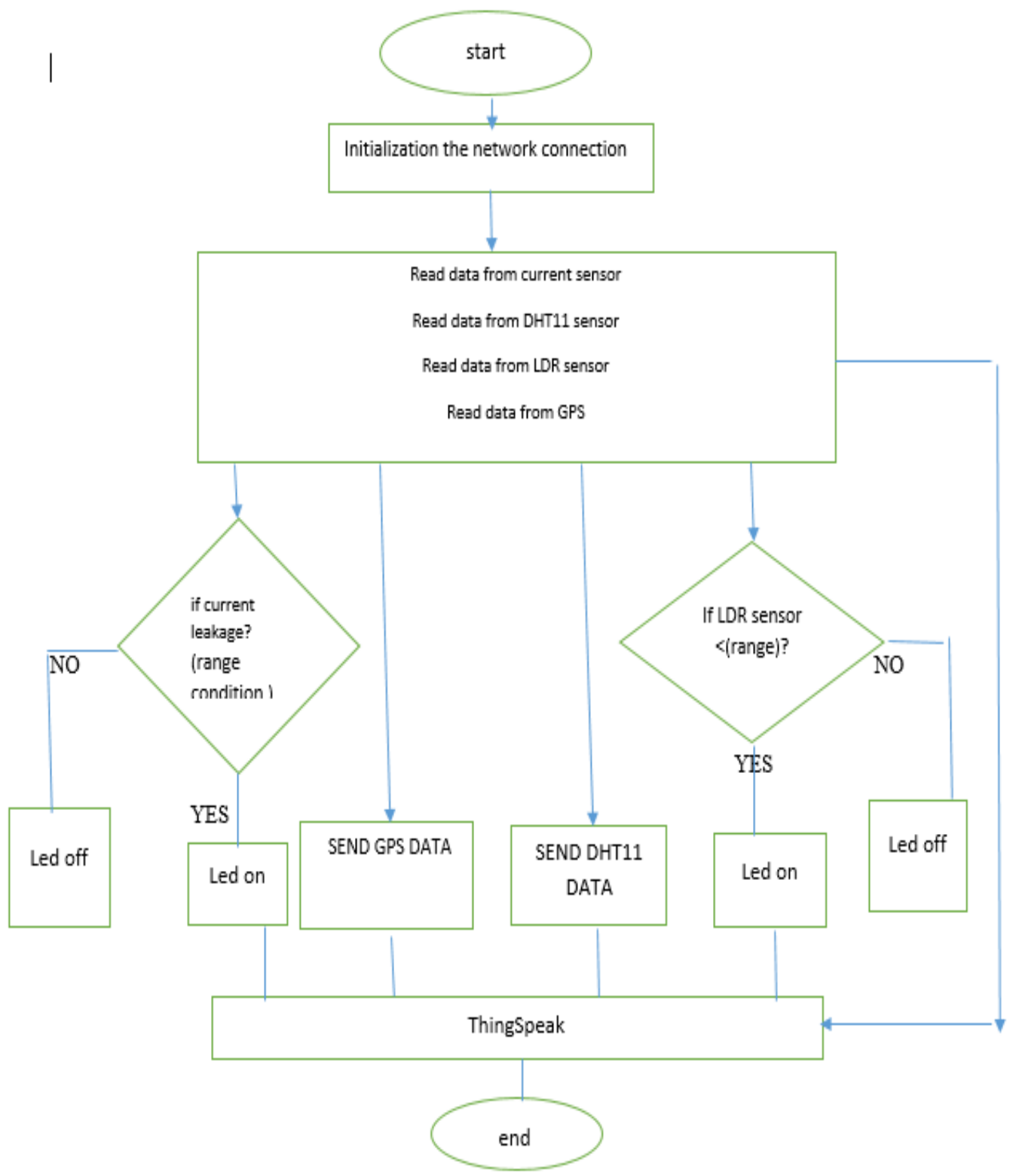

Fig. 5: System flowchart.

And the previous flowchart shows the work followed in the proposed system.

Then we need to create an account inside the API ThingSpeak, which contains the channels that are the storage location for the data loaded from the devices and sensors, this is done by creating an account and after logging in we will choose Channels> My Channels and then create a new channel. The channel has a unique identifier key that is used to identify the channel when reading or downloading data. After that, we defined the required libraries for the devices used in the system and we also linked the proposed system as we showed it through the block diagram in advance and we started recording the results and examined the system and its work after downloading the program to the NODE MCU We saw the results for more than once and they were giving similar results. as the DHT11 sensor that records the workplace temperature and humidity properly, as well as the light sensor, we noticed that when the brightness of the light increases the optical resistance increases, and by darkness it will follow the condition in the program and give a signal there are darkness by ON LED. the ACS712 sensor the current passing in the circuit used in the system, the GPS determines the work site properly and this results data uploaded to the site ThingSpeak, show Fig. 6. 

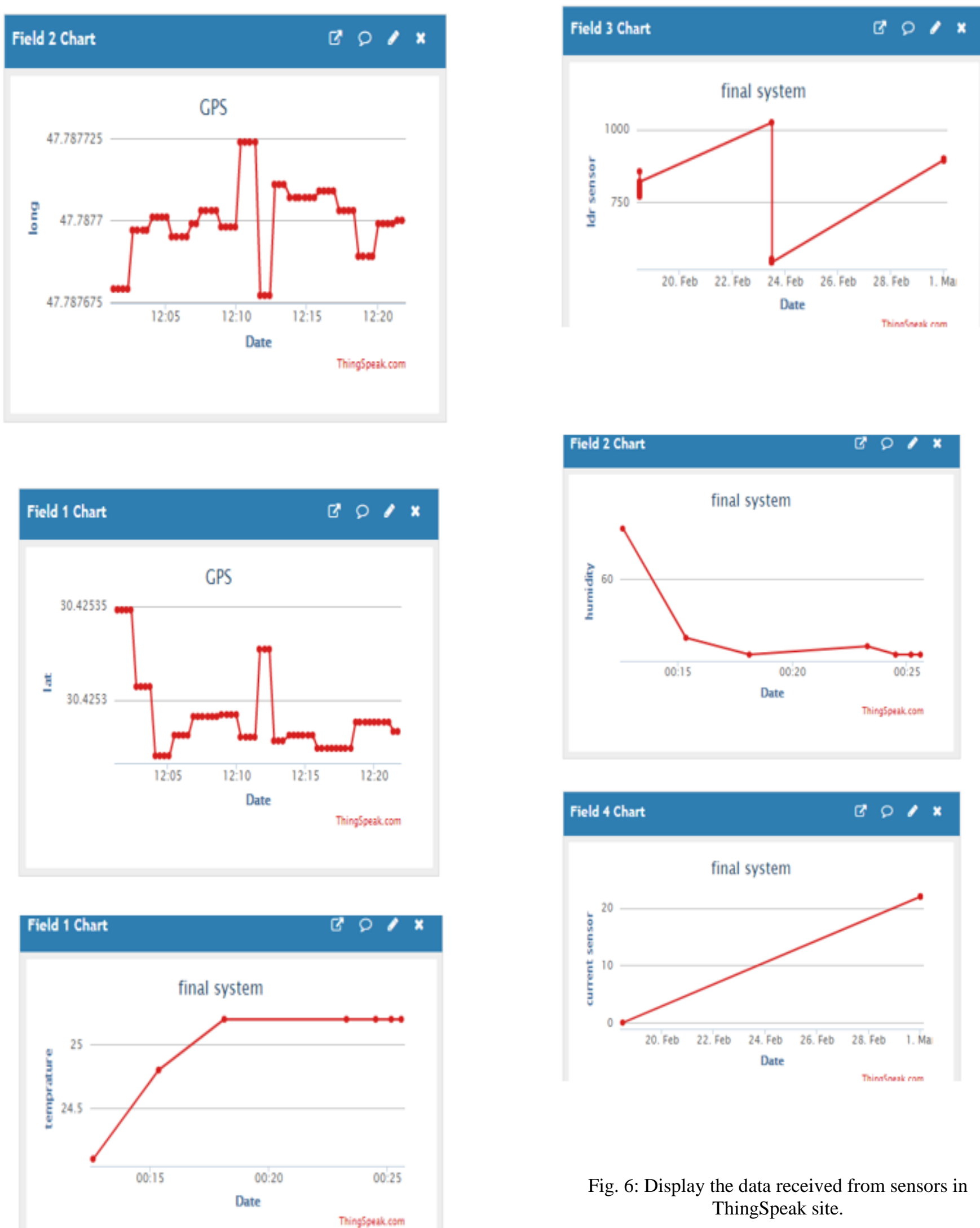

Fig. 6: Display the data received from sensors in ThingSpeak site. 


\section{CONCLUTION}

We have shown on this page what is the Internet of Things and its many uses in various fields, and here we have worked to show the status of tracking the current and the possible leakage in the electrical poles and get the location of the column by through the GPS, adding the ability to know the lighting in the column and measure the temperature and humidity of the column area and raise all these data to ThingSpeak online. This technology can be a topic to protect from the danger of the current leading to death in many places, as well as the possibilities of development at work according to the required need, which works to serve man and facilitate many of the complexities of life and the possible risk with modern technologies.

\section{CONFLICT OF INTEREST}

The authors have no conflict of relevant interest to this article.

\section{REFERENCES}

[1] Rose, Karen, Scott Eldridge, and Lyman Chapin. "The internet of things: An overview." The Internet Society (ISOC) 80 (2015).

[2] Davies, Ron. "The Internet of Things opportunities and challenges." European Parliamentary Research Service (2015).

[3] Patel, Keyur K., and Sunil M. Patel. "Internet of thingsIOT: definition, characteristics, architecture, enabling technologies, application \& future challenges." International journal of engineering science and computing 6, no. 5 (2016).

[4] Suresh, Dalli Sai, and Sivah Akash. "Implementation of Home Automation with Thingspeak Cloud."

[5] Gurjar, A. A., and Neha A. Sarnaik. "Heart attack detection by heartbeat sensing using Internet of Things: IoT." Heart 5, no. 03 (2018).

[6] Shirode, Mourvika, Monika Adaling, Jyoti Biradar, and Trupti Mate. "IOT based water quality monitoring system." Int. J. Sci. Res. Comput. Sci. Eng. Inf. Technol 3, no. 1 (2018): 5447-5454.

[7] Hidayatullah, N. A., A. C. Kurniawan, and Akhtar Kalam. "Power Transmission and Distribution Monitoring using Internet of Things (IoT) for Smart Grid." In IOP Conference Series: Materials Science and Engineering, vol. 384, no. 1, p. 012039. IOP Publishing, 2018.

[8] Memon, Azam Rafique, Bhawani Shankar Chowdhry, Syed M. Shehram Shah, Tarique Rafique Memon, and Syed MZ Abbas Shah. "An electronic information desk system for information dissemination in educational institutions."
In 2015 2nd International Conference on Computing for Sustainable Global Development (INDIACom), pp. 12751280. IEEE, 2015.

[9] ACS712, Datasheet. "Fully Integrated, Hall Effect-Based Linear Current Sensor IC with 2.1 kVRMS Isolation and a Low-Resistance Current Conductor." (2006). www.allegromicro.com

[10] www.u-blox.com. NEO-6 u-blox 6 GPS Modules Data Sheet.

[11] Ome, Nerella, and G. S. Rao. "Internet of Things (IoT) based Sensors to Cloud system using ESP8266 and Arduino Due." International Journal of Advanced Research in Computer and Communication Engineering 5, no. 10 (2016): 337-343.

[12] Zailan, Fatin Syamimi. "IMPLEMENTATION OF HOME AUTOMATION USING WIRELESS COMMUNICATION." IRC, 2016.

[13] https://www.adafruit.com/product/386

[14] Dangi, Nagendra. "Monitoring environmental parameters: humidity and temperature using Arduino based microcontroller and sensors: Microcontroller based building monitoring system." (2018).

[15] GHOSH, KUNDAN. "Globally controlled multiple relays using NODE MCU." $\mathrm{PhD}$ diss., University of Technology, 2018.

[16] Maureira, Marcello A. Gómez, Daan Olden of, and Livia Teernstra. "ThingSpeak-an API and Web Service for the Internet of Things." World Wide Web (2011).

[17] Mohamad, Auday AH, Noor Kareem Jumaa, and Sameer Hameed Majeed. "ThingSpeak Cloud Computing Platform Based ECG Diagnose System." International Journal of Computing and Digital Systems 8, no. 01 (2019): 11-18. 